\title{
Review
}

\section{Mdm2-mediated ubiquitylation: p53 and beyond}

\author{
J-C Marine ${ }^{\star, 1}$ and G Lozano ${ }^{2}$
}

The really interesting genes (RING)-finger-containing oncoprotein, Mdm2, is a promising drug target for cancer therapy. A key Mdm2 function is to promote ubiquitylation and proteasomal-dependent degradation of the tumor suppressor protein p53. Recent reports provide novel important insights into Mdm2-mediated regulation of p53 and how the physical and functional interactions between these two proteins are regulated. Moreover, a p53-independent role of Mdm2 has recently been confirmed by genetic data. These advances and their potential implications for the development of new cancer therapeutic strategies form the focus of this review.

Cell Death and Differentiation (2010) 17, 93-102; doi:10.1038/cdd.2009.68; published online 5 June 2009

Mdm2 is a key regulator of a variety of fundamental cellular processes and a very promising drug target for cancer therapy. It belongs to a large family of (really interesting gene) RING-finger-containing proteins and, as most of its other members, Mdm2 functions mainly, if not exclusively, as an E3 ligase. ${ }^{1}$ It targets various substrates for mono- and/or poly-ubiquitylation thereby regulating their activities; for instance by controlling their localization, and/or levels by proteasome-dependent degradation.

The widespread focus on Mdm2 can be attributed to its ability to bind and quench the activity of the tumor suppressor protein $553 .^{2}$ Transfection studies show that Mdm2 antagonizes $\mathrm{p} 53$ by promoting ubiquitylation and proteasomedependent degradation of p53..$^{3-5}$ MDM2 (also known as $H d m 2$ ) is amplified in about a third of human sarcomas that retained wild-type $p 53^{6}$ suggesting that overexpression of Mdm2 represents one way by which the cell inactivates p53 in the process of tumor formation. Little is known regarding other mechanisms that upregulate Mdm2 in tumors; high Mdm2 levels are indeed seen in many tumors that do not show gene amplification (Evans et al. ${ }^{7}$ and references therein).

Moreover, ample evidence suggests that Mdm2 is engaged in a complex network of regulatory interactions and thus exerts multiple p53-independent functions. Among its putative targets are several proteins playing fundamental roles in the control of cell proliferation, cell fate determination, DNA repair and other processes that may also contribute to its oncogenic potential. $^{8}$

New biochemical and genetic data have recently provided important insights into Mdm2-mediated control of p53 and how the physical and functional interactions between these two proteins are regulated in response to various signals. Additional evidence for p53-independent function of Mdm2 has also emerged from recent genetic studies. These advances and their potential implications for the development of new cancer therapeutic strategies form the focus of this review. For a more detailed discussion of Mdm2 and its various functions an interested reader should also consult references $^{9-12}$.

\section{The p53-Mdm2 Regulatory Feedback Loop}

p53 is a transcription factor that is activated by diverse genotoxic and cytotoxic stresses. Upon activation, p53 prevents the proliferation of genetically compromised cells by regulating the expression of a battery of genes that initiate cell cycle arrest, apoptosis and DNA repair. p53 also binds to two adjacent p53-responsive elements located within the Mdm2 gene to promote its transcription. ${ }^{13,14}$ Because of the antagonistic action of Mdm2 toward p53, a negative feedback loop is set up, whereby p53 transcriptionally activates Mdm2, which in turn targets p53 for degradation (Figure 1). This feedback loop is likely the key mechanism for restraining p53 activity in normal cells, in the absence of stress.

In response to stress, a decrease in Mdm2 protein levels and/or its activity and the interaction between Mdm2 and p53 lead to p53 stabilization. The mechanisms by which p53 escapes the detrimental effects of Mdm2 binding (see below for more details) vary depending on the type of stress signals. The increase in p53 levels and in transcriptional activity of p53 leads in turn to increased production of Mdm2 (Figure 1). Elegant quantitative studies of p53 show that while an individual cell may have only one pulse of p53 activity, its neighbor might have several repeated pulses. ${ }^{15}$ As the amount of radiation increases, the percentage of cells showing a high number of p53 pulses also increases.

\footnotetext{
${ }^{1}$ Laboratory For Molecular Cancer Biology, VIB-UGent, Ghent B-9052, Belgium and ${ }^{2}$ Department of Genetics, The University of Texas MD Anderson Cancer Center, The University of Texas Graduate School of Biomedical Sciences, Houston, TX 77030, USA

${ }^{*}$ Corresponding author: J-C Marine, Laboratory For Molecular Cancer Biology, VIB, Flanders Interuniversity Institute for Biotechnology, 927 Technologiepark, Ghent B-9052, Belgium. Tel: + 32933136 40; Fax: + 32933135 16; E-mail: chris.marine@dmbr.ugent.be

Keywords: MDM2; p53; ubiquitylation; cancer therapy

Abbreviations: ER, eostrogen receptor; IR, ionizing radiation; KO, knock-out; DKO, double knock-out; MEFs, mouse embryonic fibroblasts; NES, nuclear export signal; RING, really interesting genes

Received 11.2.09; revised 02.4.09; accepted 22.4.09; Edited by G Melino; published online 05.6.09
} 

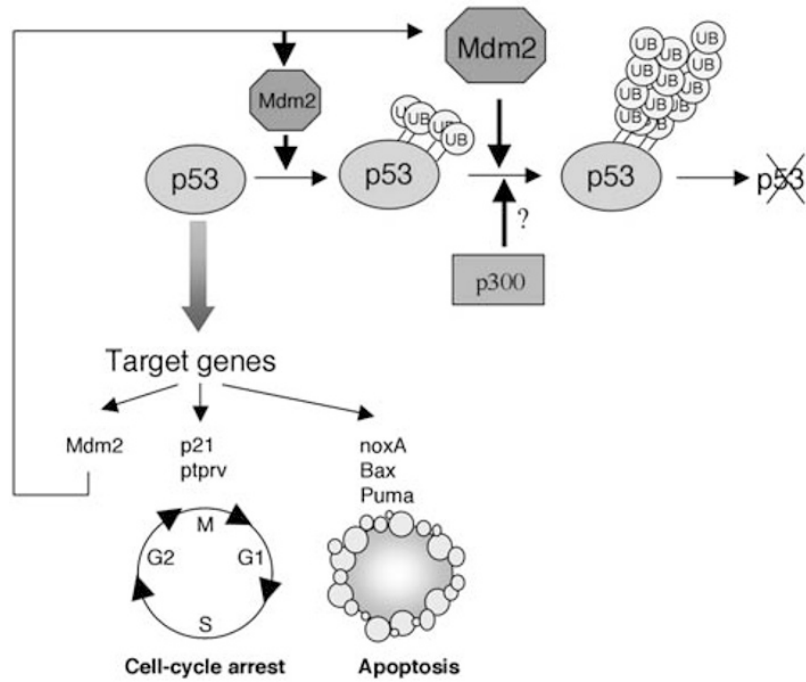

Figure 1 The p53-Mdm2 negative feedback loop

Interestingly, the mean height and width of a pulse is constant and independent of the damage level. Sister cells continue to oscillate in a correlated way even after cell division. ${ }^{16}$ These intriguing observations open new questions regarding the mechanism and function of p53 oscillatory dynamics, including the reason for the observed variation between cells. ${ }^{17}$ Understanding why some cells respond poorly is of critical importance as these genetically unstable cells will continue to proliferate and eventually become the target of additional oncogenic mutations and the cell-of-origin of tumor development.

In addition to its ability to degrade p53, Mdm2 can also antagonize $\mathrm{p} 53$ by concealing its transactivation domain. It achieves this by binding to an $\mathrm{N}$-terminal region of p53 thereby blocking its ability to associate with the transcriptional machinery. ${ }^{18,19}$ A chromatin-associated and transcriptionally incompetent p53-Mdm2 complex has been detected in cancer cell lines. ${ }^{20,21} \mathrm{Mdm} 2$ association with p53-responsive elements is reduced when p53-induced transcription is activated by DNA damage, suggesting that Mdm2 transiently localizes to p53-target genes and that a pool of latent p53 is a consequence of the recruitment of Mdm2 to chromatin. Further data in support of a role for Mdm2 in transcriptional repression was recently presented by Tang et al. ${ }^{22}$ who showed that acetylation of p53 blocks the recruitment of Mdm2 to p53-responsive promoters, a step that is critical for p53 activation of function. Mdm2 was also reported to mediate NEDD8 conjugation of p53. NEDD8 is a ubiquitin-like molecule that when attached to p53 inhibits its transcriptional activity. ${ }^{23}$ It should be noted, however, that the contribution of Mdm2 to the regulation of p53 transcriptional activity per se remains highly controversial, in particular, in light of recent genetic data that will be discussed below.

\section{Genetic Evidence for a Key Role of Mdm2 in Regulating p53 Levels}

The critical nature of the relationship between p53 and Mdm2 was determined genetically. Mdm2-null mice are lethal early in embryogenesis, but survive upon concomitant deletion of p53 suggesting constitutive activation of p53 in the absence of Mdm2. ${ }^{24,25}$ The p53-mdm2 double null mice however succumb to a tumor phenotype because of the absence of p53. In fact, p53-Mdm2 double null mice have indistinguishable survival curves and tumor spectra as p53-null mice further underscoring the importance of the p53-Mdm2 relationship. ${ }^{26}$

Cell type and tissue specificity of the p53-Mdm2 interaction was subsequently examined using conditional alleles of Mdm2. ${ }^{27}$ Loss of Mdm2 in neuronal and erythroid progenitor cells, post-mitotic neurons, smooth muscle cells and in the heart bring about cell death which is rescued by deletion of p53. ${ }^{28-32}$ Even cells that no longer have the capacity to proliferate die, and this cell death becomes catastrophic to the organism. Deletion of $M d m 2$ in the intestine also brings about cell death, although the dynamic and highly proliferative nature of the intestine compensates to allow survival of cells that retained Mdm2. ${ }^{33}$ Finally in all cases, Mdm2 loss resulted in readily detectable levels of p53 suggesting stabilization and activation of p53. Activation of p53 was further defined in some cases by measuring the levels of $p 21 \mathrm{mRNA}$ and other p53 targets. ${ }^{28,29,31,33}$ Thus, genetic studies show that Mdm2 is required to maintain the p53 protein in check at low levels in embryonic and adult tissues consistent with its ability to promote p53 degradation.

Independent studies using a p53-ER ${ }^{\text {TAM }}$ fusion protein show that the induction of p53 activity with $4-\mathrm{OH}$ tamoxifen in an Mdm2-null background yielded lethal pathologies within 5-6 days. ${ }^{34}$ Radiosensitive tissues such as bone marrow, thymus, spleen, small intestine and colon were ablated because of apoptosis. Instead, cell cycle arrest was induced in the liver and testes, exemplifying the tissue-specific nature of the p53 response. Interestingly, this model provided evidence for Mdm2-independent degradation of p53. p53ER stability was examined in the extracts derived from the spleens of these knock-in mice with and without Mdm2. Upon functional restoration of p53-ER, its levels decreased, demonstrating a functional negative feedback loop even in the absence of Mdm2. However, the delay in degradation of p53 in the absence of Mdm2 further supports the key contribution of Mdm2 to p53 degradation in vivo. The observed downregulation of p53-ER levels in the absence of Mdm2 might be under the control of other p53-regulated E3 ubiquitin ligases. Candidates for this activity include Cop1, Pirh2 and ARF-BP1, ${ }^{35}$ Topors, ${ }^{36}$ CARPs $^{37}$ or Synoviolin, ${ }^{38}$ although the role for these proteins in the regulation of p53 levels requires further genetic confirmation in vivo.

The contribution of Mdm2 to the regulation of p53 transcriptional activity is controversial. In contrast to several transfection studies, genetic studies fail to support its role in the regulation of $p 53$ transcriptional activity per se $\left({ }^{27,29,39}\right.$ for a detailed discussion). More recently, homozygous mice bearing a single-residue substitution (C462A) abolishing the E3 function without affecting p53-binding have been generated. These mice die before E7.5, and deletion of p53 rescued this lethality ${ }^{40}$ suggesting that the Mdm2-p53 physical interaction, without Mdm2-mediated p53 ubiquitylation, cannot control p53 activity sufficiently to allow early mouse embryonic development. Together, these genetic data 
strongly argue that the primary physiological function of Mdm2 is to promote p53 degradation. However, interpretation of these data is complicated by the observation that the E3 ligase activity of Mdm2 is broadly required for its ability to enforce transcriptional repression, presumably by ubiquitylation of other proteins beyond p53. ${ }^{41}$ This report indeed suggests that, when recruited to chromatin by p53-binding, Mdm2 might ubiquitylate adjacent chromatin proteins to elicit transcriptional repression, an activity that is expected to be lost in the C462A mutant.

The above genetic experiments also clearly defined p53 as an important target of Mdm2 in cell survival and in development. The importance of the p53-Mdm2 relationship to a tumor phenotype has also been demonstrated genetically. Mice with approximately $30 \%$ the levels of $M d m 2$ and mice haploinsufficient for $M d m 2$ are exquisitely sensitive to ionizing radiation (IR). ${ }^{42,43}$ These mice die within 2 weeks after treatment with a sub-lethal dose of IR. This phenotype is p53-dependent and indicates that the balance between p53 and Mdm2 levels is also critical for cell survival in response to DNA damage.

More importantly, decreased levels of Mdm2 alter the rate of tumor formation. The development of $\mathrm{E} \mu$-myc-driven B-cell lymphomas and intestinal adenomas as a result of APC loss are delayed in mice with decreased levels of Mdm2. ${ }^{44,45}$ These data from mouse models are consistent with the human genetic data. Patients with p53 mutations that also inherit an Mdm2 polymorphism slightly increasing Mdm2 levels show a statistically significant earlier age of tumor onset. ${ }^{46-48}$ These data indicate that even modest changes in Mdm2 levels, as the result of polymorphisms for example, cause measurable perturbation of p53 function and eventually cancer-related phenotypical manifestations (reviewed by Whibley et al. ${ }^{49}$ ). All in all, the balance between p53 and Mdm2 levels determines cell survival or tumorigenesis: too little Mdm2 in a wild-type p53 background kills the cell, whereas too much Mdm2 inhibits tumor suppressive functions of p53.

\section{Mdm2-Mediated p53 Ubiquitylation}

Deletion and mutational analyses have established the importance of a cluster of lysine residues within the C-terminus of p53 for Mdm2-mediated degradation. 50,51 However, homozygous mice in which seven C-terminal lysines have been changed to arginine (p53(7KR)) are viable and phenotypically normal. ${ }^{52}$ The $\mathrm{p} 53(7 \mathrm{KR})$ protein has a normal half-life and functions by and large similar to wild-type p53 in cell cycle arrest and apoptosis. Ubiquitylation of the 7KR mutant can be detected upon co-transfection with $\mathrm{Mdm} 2$; however, the ubiquitylation pattern of p53(7KR) is reduced and qualitatively different from wild-type p53. ${ }^{52}$ These data are consistent with the view that the C-terminal lysines are preferred or more accessible to ubiquitylation but that additional and/or alternative ubiquitin acceptor lysines might be present. Given that Mdm2 is itself ubiquitylated (see below), another intriguing possibility is that a mere association of ubiquitylated Mdm2 with p53 may be sufficient for the degradation of the entire complex by the proteasome. Some proteins can indeed be degraded by the proteasome without a requirement for their own ubiquitylation. ${ }^{53,54}$ Along these lines, Mdm2 may also simply shuttle p53 to the proteasome, acting as a bridging molecule between p53 and the proteasome. This notion is supported by recent observations suggesting that Mdm2 directly interacts with several components of the $26 \mathrm{~S}$ proteasome.

The ability of Mdm2 to promote p53 degradation largely depends on the integrity of the $\mathrm{N}$-terminal p53-binding domain, central acidic domain ${ }^{55-57}$ and C-terminal RINGfinger domain. ${ }^{58}$ The extreme C-terminal tail of Mdm2, which is critical for Mdm2 oligomerization, is also crucial for p53 degradation. ${ }^{59,60}$ Deletion and mutational studies, together with conformational analysis of the solution structure of the Mdm2 RING domain ${ }^{61}$ are consistent with a model in which Mdm2 forms an oligomeric complex in which the C-terminus of one molecule is brought into close proximity with the RING domain of another Mdm2. The cross-interaction between the RING and tail domains of MDM2 is required to activate the E3 activity and degradation of p53, possibly by allowing E2 binding. ${ }^{59,60}$ In addition, the C-terminal oligomerization domain of p53 is necessary for Mdm2 binding and efficient Mdm2-mediated ubiquitylation and degradation. ${ }^{62,63}$ It therefore appears that Mdm2-mediated ubiquitylation of p53 requires the formation of high molecular weight complexes containing several Mdm2 and p53 molecules. Mdmx (also known as Mdm4), a protein closely related to Mdm2, is likely another important functional component of these complexes. The RING domain of Mdmx does not possess intrinsic E3ligase activity. Instead, Mdmx may regulate p53 abundance by modulating the levels and activity of Mdm2. Dimerization, mediated by the conserved C-terminal RING domains of both Mdm2 and Mdmx, is critical to this activity. The recent crystal structure of the Mdm2/Mdmx RING domain heterodimer mapped residues required for functional interaction with the $\mathrm{E} 2, \mathrm{UbcH} 5 \mathrm{~b}$. In both $\mathrm{Mdm} 2$ and $\mathrm{Mdmx}$, residues C-terminal to the RING domain have a key role in dimer formation. In addition, these residues are part of an extended surface that is essential for ubiquitylation in trans. This study, therefore, provides a molecular basis for understanding how heterodimer formation leads to the stabilization of Mdm2, yet degradation of $p 53 .^{64}$

A covalent attachment of a polyubiquitin chain composed of at least four ubiquitin molecules onto a target lysine residue is a prerequisite of efficient proteasomal-dependent degradation. ${ }^{65}$ In contrast monoubiquitylation is involved in a number of degradation-independent processes, including endocytosis, virus budding, DNA repair and transcriptional regulation. ${ }^{66}$ Transfection studies suggest that Mdm2 promotes polyubiquitination and degradation of p53 when expressed at high levels and monoubiquitylation and nuclear export of p53 when expressed at low levels. ${ }^{67}$ Monoubiquitylation would favor nuclear export by unmasking an intrinsic nuclear export signal (NES) as a result of dissociation of oligomerized $p 53 .^{68}$ This model has however been challenged by recent data suggesting that ubiquitylation has no significant effect on the tetramerization/oligomerization of p53. ${ }^{69}$ The authors also propose that ubiquitylation-mediated repression of p53 by Mdm2 acts at least in part by inhibiting the sequence-specific DNA-binding activity of p53. A p53-ubiquitin fusion protein was recently used in an elegant study to show that ubiquitylation actually contributes to two steps before export: 
exposure of a C-terminal NES and dissociation of Mdm2. ${ }^{70}$ Monoubiquitylation directly promotes further modifications of p53 with ubiquitin-like proteins, and Mdm2 promotes the interaction of the SUMO E3 ligase PIASy with p53, enhancing both sumoylation and nuclear export. ${ }^{70}$

Mdm2 may also function as a chaperone-like molecule for p53 and this function appears essential for ubiquitylation and export of p53. Recent data suggest that wild-type p53 undergoes an Mdm2-dependent conformational change before it is degraded by the proteasome. Notably, this conformational change was opposed by the heat-shock protein HSP90 and did not require the Mdm2-RING domain and p53 ubiquitination. ${ }^{71}$ The conformational change precedes p53 ubiquitylation and the addition of ubiquitin to the C-terminal lysines and DNA-binding domain of p53 would then promote the nuclear export of $\mathrm{p} 53 .^{72}$

Polyubiquitin chains can be conjugated to the same lysines of p53 either by Mdm2, when it is expressed at high levels, for example, as it occurs in response to activation of p53 (see below and Figure 1) and in some tumor cells, or by another E4-like enzyme. One report indicates that p300 shows intrinsic ubiquitin ligase activity and cooperates with Mdm2 in catalyzing p53 polyubiquitylation and subsequent proteasomal-dependent degradation ${ }^{73}$ (Figure 1). Surprisingly, however, p300 does not posses an E3 ligase domain. It therefore remains to be firmly established whether the reported intrinsic ubiquitin ligase activity is mediated by p300 itself or by another coimmunoprecipitating protein.

\section{Regulation of Mdm2-Mediated p53 Ubiquitylation}

Numerous mechanisms regulate Mdm2-directed p53 ubiquitylation (reviewed by Michael and Oren ${ }^{10}$; Brooks and $\mathrm{Gu}^{35}$ ). For example, DNA damaging agents, such as ionizing radiation or ultra violet, bring about posttranslational modifications of both Mdm2 and p53, in some cases leading to a reduction of the affinity of $\mathrm{p} 53$ to Mdm2. Some modifications, such as phosphorylation or acetylation, directly reduce the ability of Mdm2 to promote p53 ubiquitylation. The simplest and perhaps the most efficient way to regulate Mdm2mediated ubiquitylation involves downregulation of its expression, for example through the regulation of transcriptional rate or half-lives of the transcript and/or the protein. Interaction with other cellular factors can also play a regulatory role. The tumor suppressor ARF and oncoprotein Mdmx are the two best-studied examples (reviewed by Sherr $^{74}$ and Marine et al. ${ }^{75}$ ) (Figure 2). Both proteins bind Mdm2 tightly and affect its cellular localization, stability and activity through a variety of mechanisms that are yet to be fully elucidated.

All these mechanisms likely cooperate to impose a tight and fine-tuned regulation on p53 activity. Moreover, the list of new mechanisms and regulators reported to affect the Mdm2-p53 loop is growing rapidly. However, as the p53-Mdm2 loop is exquisitely sensitive to a variety of small experimental parameters that are often difficult to control, such as oxygen concentrations, growth factor exposure and extent of DNA damage, great care should be taken when interpreting this flurry of data, especially those from tissue culture work. Owing to space limitation, we only focus below on a handful of examples that illustrate the complexity of this regulation.

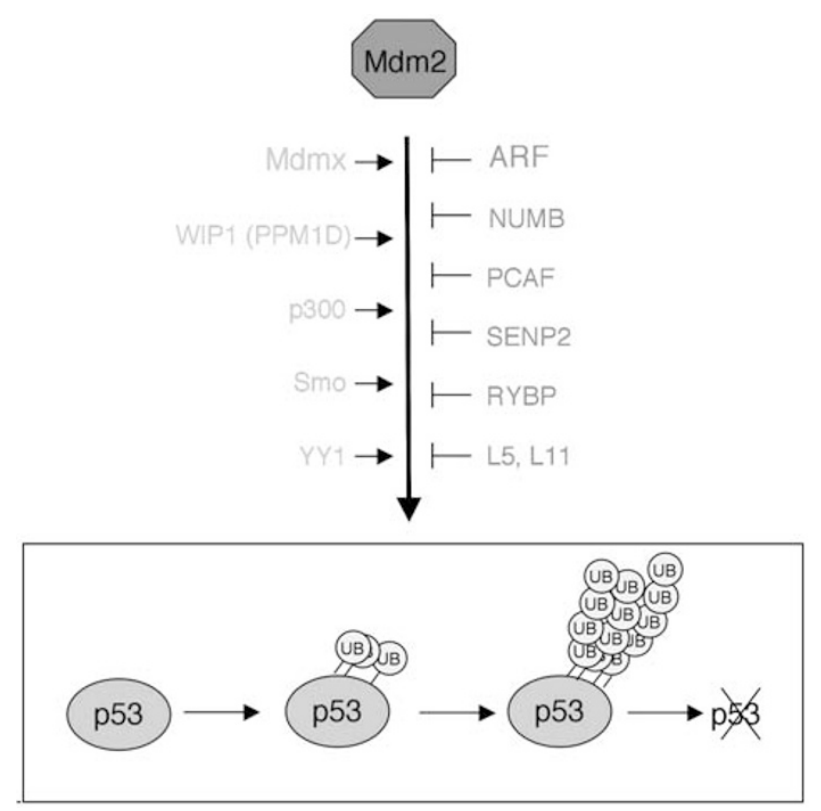

Figure 2 A non-comprehensive list of recently described regulators of Mdm2mediated p53 ubiquitylation and degradation

Posttranslational modifications. In response to low dose of IR, p53 activation often results in a transient cell cycle arrest. When the damage has been repaired, restoration of low, pre-stress levels of p53 must be achieved before the cell cycle can resume. Phosphatase Wip1 (also known as PPM1D) was recently identified as a key component of the p53-Mdm2 negative feedback loop ${ }^{76}$ (Figure 2). Wip1 is a transcriptional target of $\mathrm{p} 53^{77,78}$ that catalyzes dephosphorylation of Mdm2 at Ser-395, resulting in Mdm2 stabilization, enhanced Mdm2-p53 binding and subsequent ubiquitylation. ${ }^{76}$ Ser-395 is a target of the DNA-damageinduced kinase ATM, which participates in Mdm2 degradation in response to IR. ${ }^{79}$ The data suggest that Wip1 facilitates Mdm2-mediated degradation of p53 by counteracting DNA-damage-induced Mdm2 degradation. When overexpressed Wip1 may function as an oncogene; indeed, it is amplified and overexpressed in breast cancers. ${ }^{80}$ The involvement of Wip1 as a key gatekeeper in the p53/Mdm2 loop is further highlighted by its role in maintaining the uniform shape of $\mathrm{p} 53$ pulses in response to persistent DNA damage. ${ }^{81}$

The hedgehog $(\mathrm{Hh})$ signaling pathway plays an important role in organogenesis during normal development but the pathway is also frequently activated in human cancers. Its role in cancer development involves, at least partially, the Mdm2p53 hub. ${ }^{82}$ Constitutively active mutants of Smoothened (Smo), a transducer of the Hh signaling pathway, inhibit the accumulation of p53 in several human cancer cell lines by facilitating the Mdm2-p53 physical interaction and promoting p53 ubiquitylation. Hh signaling induces phosphorylation of Mdm2 on Serines 166 and 186, known activating sites for Mdm2. Importantly, mutations in Smo enhance the proliferation of mouse embryonic fibroblasts (MEFs), partially inhibit p53-dependent apoptosis and reduce cell growth inhibition in oncogene-expressing MEFs. Taken together, the Hh pathway 
seems to affect oncogenesis by enhancing Mdm2 activity and thereby inhibiting p53 tumor suppression function (Figure 2).

Beside phosphorylation, Mdm2 is subject to other posttranslational modifications, including acetylation, ubiquitination (see below) and sumoylation. ${ }^{83}$ The SUMO-specific protease 2 (SENP2) acts by removing the ubiquitin-like molecule, SUMO, from its substrate. Recent genetic data suggest that SENP2 targets Mdm2 sumoylation and thus regulates its E3 ligase activity. ${ }^{84}$ Targeted disruption of SENP2 in mice affects the expansion of trophoblast progenitors and their maturation, a phenotype that is alleviated upon p53 downregulation. Reintroduction of SENP2 into these mutants reduces sumoylation of Mdm2, diminishes p53 levels and rescues trophoblast development. These data therefore suggest that sumoylation plays a key role in the control of Mdm2 E3 ligase activity (Figure 2).

Direct binding to Mdm2 - trimeric complexes. Numb controls cell fate choices by antagonizing the activity of the plasma membrane receptor of the NOTCH family. ${ }^{85} \mathrm{Mdm} 2$ had been shown to bind NUMB and promote its ubiquitylation and degradation. ${ }^{86,87}$ The NUMB-Mdm2 physical and functional interaction has more recently been revisited. The physical interaction has been confirmed at endogenous levels of protein expression. ${ }^{88}$ Sequential coimmunoprecipitation assays further demonstrate the existence of a p53-Mdm2-Numb trimeric complex in cells, whereby Numb prevents Mdm2-mediated p53 ubiquitylation and subsequent degradation (Figure 2). The ability of purified recombinant NUMB to interfere with Mdm2-dependent p53 ubiquitylation was confirmed in in vitro ubiquitylation assays. Moreover, p53 half-life, steady-state protein levels and activity are significantly reduced in NUMB knockdown cells. Consistently, NUMB expression is frequently lost in breast cancers and is associated with decreased p53 levels and increased chemoresistance. ${ }^{88}$ Loss of NUMB expression also enhances the activity of the oncogenic receptor $\mathrm{NOTCH}$. Hence, a single event - loss of NUMB expression - leads to an activation of an oncogene and attenuation of the p53 tumor suppressor pathway. Biologically, this alteration results in an aggressive tumor phenotype, as highlighted by the observation that NUMB-defective breast cancers display poor prognosis. The study raises a number of questions, one of which is the issue of how NUMB a cytoplasmic protein, mainly associated with biomembranes, can affect the actions of p53 and Mdm2, most of which are nuclear. Nevertheless, given the role of NUMB in binary cell fate decisions, this study suggests a role for $\mathrm{p} 53$ in the control of asymmetric cell division. Such a function for p53 has been suggested earlier ${ }^{89}$ but this study may help revisit this important issue. The proposed model predicts that inactivation of the p53Mdm2-NUMB axis, as the result of decreased NUMB expression for instance, may cause the skewing of stem cell division toward a symmetric pattern and thus favor tumor development.

The Yin Yang 1 (YY1) transcription factor plays an essential role in development (as demonstrated by the consequence of genetic ablation of its expression in the mouse), possibly as the result of decreased p53 ubiquitylation, p53 protein accumulation and activation of its function. ${ }^{90}$ Overexpression of YY1 stimulated p53 ubiquitylation and degradation, and the recombinant $Y Y 1$ was sufficient to induce Mdm2-mediated p53 polyubiquitination in vitro. Direct physical interactions between $Y Y 1$ with Mdm2 and p53 have been demonstrated suggesting that $Y Y 1$ regulates p53 ubiquitylation by facilitating the p53-Mdm2 interaction. The data therefore point to YY1 as a potential cofactor for Mdm2 in the regulation of p53 homeostasis (Figure 2).

In contrast, the protein RYBP (RING1- and YY1-binding protein), a member of the polycomb group (PcG) decreases Mdm2-mediated p53 ubiquitylation upon binding to Mdm2. ${ }^{91}$ RYBP induces cell cycle arrest by stabilizing and activating p53. Accordingly, RYBP expression is decreased in human cancers, suggesting that it exhibits tumor suppressor activity owing to its ability to regulate the p53-Mdm2 loop (Figure 2).

Regulation of Mdm2 stability. Mdm2 is a very short-lived protein, whose rapid degradation is due to ubiquitindependent proteolysis. ${ }^{92}$ In fact, in addition to ubiquitylating p53, Mdm2 can also drive its own ubiquitination. ${ }^{58,93}$ This auto-ubiquitylation ability can be separated from its ability to ubiquitylate p53; in fact, some forms of genotoxic damage stabilize p53 by promoting the auto-degradation of Mdm2, thereby shifting the balance between the levels of the two proteins. ${ }^{94}$ Recent genetic data suggest, however, that endogenous Mdm2 does not regulate its own stability by self-ubiquitylation. ${ }^{95} \mathrm{Mdm} 2$ steady-state levels observed in mice in which the RING E3 ubiquitin ligase activity of Mdm2 was abrogated by a single-point mutation were comparable with the levels in wild-type mice, implying that Mdm2 stability is controlled by another E3 ubiquitin ligase in vivo. The histone acetyltransferase PCAF (p300-CBP-associated factor) has recently been proposed as a putative candidate $^{96}$ as knockdown of PCAF in U2OS and HeLa cancer cell lines stabilized Mdm2. PCAF possesses an intrinsic ubiquitylation activity that is critical for controlling Mdm2 stability, and thus p53 function.

Regulation of Mdm2 Iocalization. Mdm2-mediated p53 ubiquitylation and degradation can also be regulated by disrupting p53 binding and sequestration of Mdm2 to a specific cellular compartment. ARF-dependent sequestration of Mdm2 to the nucleoli is one of the proposed mechanisms through which ARF stabilizes p53. ${ }^{97}$ Another nucleolar protein, NPM (or B23), interacts with Mdm2 and protects p53 from Mdm2-mediated degradation. ${ }^{98}$ The PML protein interacts with $\mathrm{Mdm}^{99}$ and, similar to $\mathrm{ARF}$, enhances p53 stability by sequestering Mdm2 to the nucleolus. ${ }^{100}$ The data convincingly show that, following DNA damage, both PML and Mdm2 accumulate in the nucleoli in an ARF-independent manner. The nucleolar localization of Mdm2 was impaired in PML-deficient cells, as was p53 stabilization and the induction of apoptosis. Moreover, PML physically associates with the ribosomal protein $\mathrm{L} 11$ and this interaction is largely responsible for the recruitment of PML to the nucleoli after DNA damage. Importantly, L11, as well as other ribosomal proteins including $L 5$, directly interacts with Mdm2 and inhibits its E3 function. ${ }^{101} \mathrm{~L} 5$ and L11 may cooperate to ensure robust inhibition of the E3 activity of $\mathrm{Mdm2}$, and stabilization and activation of $\mathrm{p} 53 .{ }^{102}$ The interaction 
between the ribosomal proteins and Mdm2 may be induced under conditions of ribosomal biogenesis stress, and thus lead to a p53-dependent appropriate cellular response. Together these data further confirm a previously recognized important role for the nucleolus in the regulation of the Mdm2-p53 feedback loop. ${ }^{103}$

Other Mdm2-binding proteins acting in the p53 pathway. Mdm2 targets a number of other proteins, including Mdmx another key player in the p53 pathway. Mdmx and its role in the regulation of the p53-Mdm2 pathway have recently been discussed elsewhere. ${ }^{104,105}$

Another Mdm2-interacting protein that can no longer be ignored is mutant p53. Knock-in mice expressing p53R175H, a mutant form of p53 frequently found in human cancers, have recently been generated and have provided in vivo evidence for the importance of the Mdm2-mutant p53 interaction. ${ }^{106}$ Cells from mice homozygous for the p53R172H mutation have normal levels of mutant p53. In the absence of Mdm2, however, p53R172H becomes stable in normal cells and leads to its gain-of-function activities in vivo. Thus, constitutive levels of Mdm2 regulate the normal levels of p53 whether it contains a mutation or not. The tissue-specific nature of mutant p53 stabilization indicates that either p53 is not important in some cell types or that other negative regulators of p53 take precedence over Mdm2 in specific cell types. ${ }^{106}$ However, as p53 can no longer activate transcription of the $M d m 2$, the negative feedback loop cannot be established; hence, upon DNA damage, mutant p53 is stabilized. ${ }^{106}$ In heterozygous mice with one mutant and one wild-type p53 allele, the increased stability of mutant p53 in response to DNA damage functions as a dominant negative and dampens wild-type p53 activity. Proliferating cells with mutant p53 that receive DNA damage signals therefore do not need to lose the wild-type p53 allele to accumulate additional alterations during tumor development.

Various transfection studies have provided existence of additional targets for Mdm2. ${ }^{8}$ Below we focus on the most recent additions to this list. Among the new putative targets are a number of proteins that act as modulators and/or downstream effectors of the p53 pathway. The JMY protein belongs to this category and functions as an essential p53 cofactor. ${ }^{107} \mathrm{Mdm} 2$ binds $\mathrm{JMY}$ and catalyze its ubiquitindependent proteasomal degradation, thereby overcoming the ability of JMY to augment p53 response. ${ }^{108}$ Similarly, Mdm2 promotes ubiquitin-dependent proteasomal degradation of hnRNP K, another p53 cofactor. ${ }^{109}$ p53 and hnRNP $\mathrm{K}$ are recruited to $p 53$-responsive promoters in a mutually dependent manner in response to DNA damage. hnRNP K protein rapidly accumulates in response to DNA damage and facilitates induction of p53-target genes and cell-cycle checkpoint arrest. hnRNP K depletion strikingly impairs p53 transcriptional activity.

HIPK2 is a kinase that phosphorylates p53 at ser-46 to promote its proapoptotic activity upon severe, non-reparable, DNA damage. ${ }^{110,111}$ Recent data suggest that HIPK2 undergoes Mdm2-mediated ubiquitylation and subsequent degradation. ${ }^{112}$ This finding suggests that p53 represses its own phosphorylation at Ser-46 as the result of its ability to induce Mdm2 expression. Thus, in cells exposed to low dose of radiation, the p53-Mdm2-HIPK2 pathway favors cell survival by inhibiting $\mathrm{p} 53$ proapoptotic activities.

\section{p53-Independent Functions of Mdm2}

Not all Mdm2 targets function in the p53 pathway. For instance, Mdm2-dependent ubiquitylation contributes to RasERK-mediated degradation of the transcription factor Forkhead box $\mathrm{O} 3 \mathrm{a}$ (FOXO3a) to promote cell proliferation. ${ }^{113}$ But the ability of Mdm2 to regulate the activity of its targets need not in all cases be through the induction of their proteasomaldependent destruction. Mdm2 promotes monoubiquitination of histones $\mathrm{H} 2 \mathrm{~A}$ and $\mathrm{H} 2 \mathrm{~B}$ by directly interacting with them. ${ }^{41}$ Endogenous Mdm2 is recruited to the p53-responsive p21 (waf1) promoter, while overexpressed Mdm2 enhances histone ubiquitylation in the vicinity of the p53-binding site within that promoter. ${ }^{41}$ Moreover, when recruited to a promoter in the absence of $\mathrm{p53}, \mathrm{Mdm} 2$ represses transcription in a manner that requires its RING domain. Hence, histone monoubiquitylation may constitute a new mechanism through which Mdm2 represses transcription. Another link between Mdm2 and epigenetic regulation and DNA metabolism is suggested by a recent report of Mdm2's ability to promote monoubiquitylation of dihydrofolate reductase. ${ }^{114}$ Mdm2, in this context, inhibits the activity of its target without affecting its steady-state level.

Tumor studies provide further evidence for the importance of p53-independent functions of Mdm2. The relationship between p53 and Mdm2 suggests that as an alternative to mutation of $p 53$, tumor cells may increase Mdm2 levels to inactivate p53. ${ }^{115}$ Many tumors including sarcomas and head and neck squamous carcinomas indeed have high levels of Mdm2 and retain a wild-type $p 53$ gene. ${ }^{116,117}$ However, this is not always the case as some tumors have high levels of Mdm2 and mutations in p53. ${ }^{118,119}$ These tumor data suggest that cells expressing high levels of Mdm2 have additional growth advantages that fuel tumorigenesis. Numerous data suggest that increased levels of Mdm2 provide a growth advantage in a p53-independent manner. For example, overexpression of Mdm2 predisposes mice to spontaneous tumor formation. ${ }^{120}$ Importantly, when also deficient for p53, these mice had an increased incidence of sarcoma formation relative to p53-null mice, suggesting p53-independent contributions to tumorigenesis. High levels of $M d m 2$ in the mammary gland lead to genomic instability independently of $p 53 .^{121}$ Perhaps newly discovered interactions of Mdm2 with $\mathrm{Nbs} 1$ account for the genomic instability. ${ }^{122}$ In the $\mathrm{E} \mu$-myc model, tumors that harbor p53 mutations also have increased levels of Mdm2. ${ }^{44}$ Interestingly, many other Mdm2-interacting proteins that have been identified play a key role in the control of cell proliferation, including $\mathrm{Rb}, \mathrm{E} 2 \mathrm{~F} 1$ and Smads. These proteins may therefore contribute at least to some extent to Mdm2 oncogenic function that is p53-independent.

What might be the physiological relevance of all these putative interactions? There has been a great deal of skepticism regarding the p53-independent functions of Mdm2, mainly because p53-Mdm2 double knock out (KO) (DKO) mice are indistinguishable from p53 KO mice. ${ }^{26}$ Some of these interactions may only become effective and relevant under conditions in which Mdm2 levels are elevated, such as 
under stress - in response to activation of the negative feedback loop - and in human tumors. As mentioned above Mdm2 expression is dramatically elevated in some tumors; under these conditions, a pool of Mdm2 may become available to target numerous other substrates, including proteins that are not directly involved in the p53 pathway.

Moreover, we may simply have not compared the p53 KO and p53-Mdm2 DKO mice in enough detail. A recently published study supports this view. ${ }^{123} \mathrm{Mdm} 2$ has previously been shown to physically associate with IGF-1R and cause its ubiquitylation in vitro. ${ }^{124}$ Consistent with the ability of Mdm2 to promote ubiquitylation and proteasome-mediated destruction of IGF-1R independently of p53, loss of Mdm2 leads to a significant increase in IGF1-R protein levels both in cells lacking and expressing $p 53 .{ }^{123}$ Interestingly, IGF-1R protects cells from DNA-damage-induced apoptosis only in the absence of Mdm2. These data therefore highlight a physiological role for Mdm2 in the control of IGF1 signaling and provide genetic evidence for a p53-independent proapoptotic function of Mdm2.

\section{Mdm2 as a Target for Cancer Therapy}

Ample evidence suggests that transformed cells are more sensitive to p53-induced apoptosis than their normal counterparts. Restoration of p53 activity in mice cause tumor-specific cell killing or induction of senescence. ${ }^{125-127}$ Activation of the p53 response becomes, therefore, an attractive therapeutic goal. In particular, the physical interaction between p53 and Mdm2 has recently become the target for the development of new cancer therapeutic strategies (Figure 3). ${ }^{128-130}$

Nutlin $3 a$ is a small molecule that binds Mdm2 in its p53binding domain and thereby disrupting the Mdm2-p53 interaction. ${ }^{129}$ A family of small molecules, HLI98, also binds Mdm2, but their binding inhibits the E3 ligase activity at the carboxyl terminus. ${ }^{130}$ Another small molecule - RITA (reactivation of p53 and induction of tumor cell apoptosis) - blocks the Mdm2-p53 interaction, but by direct interaction with p53. ${ }^{128}$ As numerous studies in mice indicate that $\mathrm{Mdm2}$ loss leads to p53-dependent pathologies in normal tissues, the toxicity of these molecules in vivo will have to be assessed. However, genetic ablation of Mdm2 is more drastic than the use of small molecule inhibitors that have a limited half-life and it is therefore possible that a therapeutic window can be found.

As mutations in p53 abolish p53 binding to DNA but leave the Mdm2-interacting domain intact, the interaction of these small molecules with mutant p53 and stabilization of the mutant p53 protein cannot be ignored. The loss of Mdm2, which mimics the inhibition of the Mdm2-p53 interaction, led to a stabilization of mutant p53 and an increased incidence of metastasis as compared with mice lacking $p 53 .{ }^{106}$ The model predicts that patients with mutations in p53 will stabilize mutant p53 upon treatment with small molecule inhibitors of Mdm2, regardless of the length of small molecule administration. In tumors with p53 loss of heterozygousity, the stabilization of mutant p53 is likely to yield a worse outcome. In tumors that retain a wild-type p53, wild-type p53 may eventually overcome the stabilization of the mutant p53. Whether the presence of one mutant p53 contributes to a metastatic outcome remains unknown. However, in mutant p53 heterozygous mice treated with IR, the mutant p53 halflife is longer than that of wild-type p53; this overall decrease in p53 activity might in turn lead to the acquisition of additional mutations. ${ }^{106}$

In light of the observations described above, small molecules inhibitors should be used in tumor cells that retain wild-type p53 whether or not Mdm2 overexpression is detected, as small differences in Mdm2 levels affect a tumor phenotype. ${ }^{44,45}$ The small molecules that bind Mdm2 may have additional efficacy as these may eliminate Mdm2dependent p53-independent activities. On the other hand, they may stabilize Mdm2 and increase its oncogenic

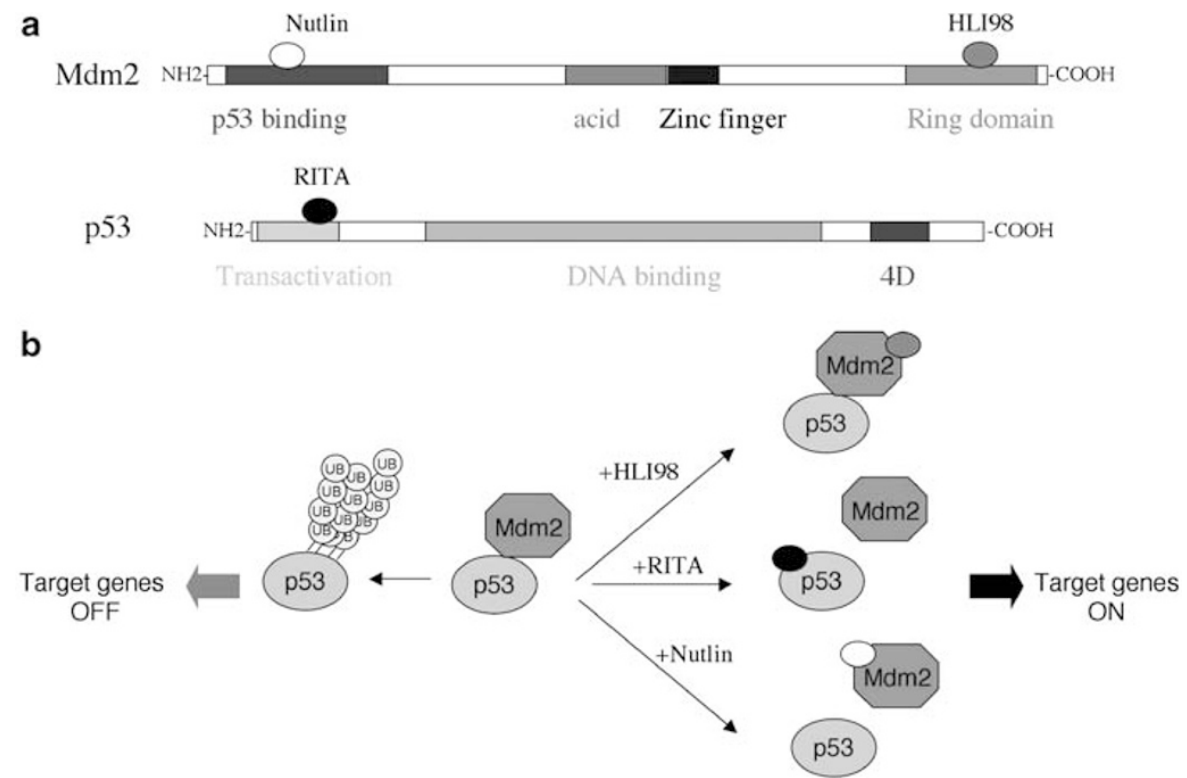

Figure 3 (a) Schematic representation of the Mdm2 and p53 proteins. (b) Mechanisms of action of the small molecules HLI98; RITA and Nutlin 
functions. Although this possibility has not been directly tested, HLI98, for example, was shown to stabilize both Mdm2 and p53 and result in p53-independent toxicity. ${ }^{130}$

Numerous activities of Mdm2 affect cell survival and tumor growth, only some of which are p53-dependent. An understanding of the Mdm2-dependent mechanisms that contribute to the maintenance of tumor growth and development are crucial to making therapeutic decisions for individual patient tumors.

1. Jackson PK, Eldridge AG, Freed E, Furstenthal L, Hsu JY, Kaiser BK et al. (2000) The lore of the RINGs: substrate recognition and catalysis by ubiquitin ligases. Trends Cell Biol 10: 429-439.

2. Momand J, Zambetti GP, Olson DC, George D, Levine AJ (1992) The mdm-2 oncogene product forms a complex with the p53 protein and inhibits p53-mediated transactivation. Cell 69: 1237-1245.

3. Haupt Y, Maya R, Kazaz A, Oren M (1997) Mdm2 promotes the rapid degradation of p53. Nature 387: 296-299.

4. Honda R, Tanaka H, Yasuda H (1997) Oncoprotein MDM2 is a ubiquitin ligase E3 for tumor suppressor p53. FEBS 420: 25-27.

5. Kubbutat MH, Jones SN, Vousden KH (1997) Regulation of p53 stability by Mdm2. Nature 387: 299-303.

6. Leach FS, Tokino T, Meltzer P, Burrell M, Oliner JD, Smith S et al. (1993) p53 Mutation and MDM2 amplification in human soft tissue sarcomas. Cancer Res 53: 2231-2234.

7. Evans SC, Viswanathan M, Grier JD, Narayana M, El-Naggar AK, Lozano G (2001) An alternatively spliced HDM2 product increases p53 activity by inhibiting HDM2. Oncogene 20: 4041-4049.

8. Ganguli G, Wasylyk B (2003) p53-independent functions of MDM2. Mol Cancer Res 1: 1027-1035.

9. Freedman DA, Wu L, Levine AJ (1999) Functions of the MDM2 oncoprotein. Cell Mol Life Sci 55: 96-107.

10. Michael D, Oren M (2003) The RING domain of Mdm2 mediates histone ubiquitylation and transcriptional repression. Cancer Biol 13: 49-58.

11. Iwakuma T, Lozano G (2003) MDM2, an introduction. Mol Cancer Res 1: 993-1000.

12. Levav-Cohen $Y$, Haupt S, Haupt $Y$ (2004) Dynamics of the p53-Mdm2 feedback loop in individual cells. Growth Factors 23: 183-192.

13. Barak Y, Juven T, Haffner R, Oren M (1993) mdm2 expression is induced by wild type p53 activity. EMBO J 12: 461-468.

14. Perry ME, Piette J, Zawadzki JA, Harvey D, Levine AJ (1993) The mdm-2 gene is induced in response to UV light in a p53-dependent manner. Proc Natl Acad Sci USA 90: 11623-11627.

15. Lahav G, Rosenfeld N, Sigal A, Geva-Zatorsky N, Levine AJ, Elowitz MB et al. (2004) Dynamics of the p53-Mdm2 feedback loop in individual cells. Nat Genet 36: 147-150.

16. Geva-Zatorsky N, Rosenfeld N, Itzkovitz S, Milo R, Sigal A, Dekel E et al. (2006) Oscillations and variability in the p53 system. Mol Syst Biol 2: 2006.0033.

17. Lahav G (2008) Oscillations by the p53-Mdm2 feedback loop. Adv Exp Med Biol 641: 28-38.

18. Oliner JD, Kinzler KW, Meltzer PS, George DL, Vogelstein B (1993) Amplification of a gene encoding a p53-associated protein in human sarcomas. Nature 362: 857-860.

19. Thut CJ, Goodrich JA, Tjian R (1997) Repression of p53-mediated transcription by MDM2: a dual mechanism. Genes Dev 11: 1974-1986.

20. Arva NC, Gopen TR, Talbott KE, Campbell LE, Chicas A, White DE et al. (2005) A chromatin-associated and transcriptionally inactive p53-Mdm2 complex occurs in mdm2 SNP309 homozygous cells. J Biol Chem 280: 26776-26787.

21. White DE, Talbott KE, Arva NC, Bargonetti J (2006) Mouse double minute 2 associates with chromatin in the presence of $\mathrm{p} 53$ and is released to facilitate activation of transcription. Cancer Res 66: 3463-3470.

22. Tang Y, Zhao W, Chen Y, Zhao Y, Gu W (2008) Acetylation is indispensable for p53 activation. Cell 133: 612-626.

23. Xirodimas DP, Saville MK, Bourdon JC, Hay RT, Lane DP (2004) Mdm2-mediated NEDD8 conjugation of $p 53$ inhibits its transcriptional activity. Cell 118: 83-97.

24. Jones SN, Roe AE, Donehower LA, Bradley A (1995) Rescue of embryonic lethality in Mdm2-deficient mice by absence of p53. Nature 378: 206-208.

25. Montes de Oca Luna R, Wagner DS, Lozano G (1995) Rescue of early embryonic lethality in mdm2-deficient mice by deletion of p53. Nature 378: 203-206.

26. McDonnell TJ, Montes de Oca Luna R, Cho S, Amelse LL, Chavez-Reyes A, Lozano G (1999) Loss of one but not two mdm2 null alleles alters the tumour spectrum in p53 null mice. J Pathol 188: 322-328.

27. Marine JC, Francoz S, Maetens M, Wahl G, Toledo F, Lozano G (2006) Keeping p53 in check: essential and synergistic functions of Mdm2 and Mdm4. Cell Death Differ 13: 927-934.

28. Xiong S, Van Pelt CS, Elizondo-Fraire AC, Liu G, Lozano G (2006) Synergistic roles of Mdm2 and Mdm4 for p53 inhibition in central nervous system development. Proc Natl Acad Sci USA 103: 3226-3231.
29. Francoz S, Froment P, Bogaerts S, De Clercq S, Maetens M, Doumont G et al. (2006) Mdm4 and Mdm2 cooperate to inhibit p53 activity in proliferating and quiescent cells in vivo. Proc Natl Acad Sci USA 103: 3232-3237.

30. Grier JD, Xiong S, Elizondo-Fraire AC, Parant JM, Lozano G (2006) Tissue-specific differences of p53 inhibition by Mdm2 and Mdm4. Mol Cell Biol 26: 192-198.

31. Boesten LS, Zadelaar SM, De Clercq S, Francoz S, van Nieuwkoop A, Biessen EA et al. (2006) Mdm2, but not Mdm4, protects terminally differentiated smooth muscle cells from p53-mediated caspase-3-independent cell death. Cell Death Differ 13: 2089-2098.

32. Maetens M, Doumont G, Clercq SD, Francoz S, Froment P, Bellefroid E et al. (2007) Distinct roles of Mdm2 and Mdm4 in red cell production. Blood 109: 2630-2633.

33. Valentin-Vega YA, Okano H, Lozano G (2008) The intestinal epithelium compensates for p53-mediated cell death and guarantees organismal survival. Cell Death Differ 15 1772-1781.

34. Ringshausen I, O'Shea CC, Finch AJ, Swigart LB, Evan GI (2006) Mdm2 is critically and continuously required to suppress lethal p53 activity in vivo. Cancer Cell 10: 501-514.

35. Brooks CL, Gu W (2006) p53 ubiquitination: Mdm2 and beyond. Mol Cell 21: 307-315.

36. Rajendra R, Malegaonkar D, Pungaliya P, Marshall H, Rasheed Z, Brownell J et al. (2004) Topors functions as an E3 ubiquitin ligase with specific E2 enzymes and ubiquitinates p53. J Biol Chem 279: 36440-36444.

37. Yang W, Rozan LM, McDonald III ER, Navaraj A, Liu JJ, Matthew EM et al. (2007) CARPs are ubiquitin ligases that promote MDM2-independent p53 and phospho-p53ser20 degradation. J Biol Chem 282: 3273-3281.

38. Yamasaki S, Yagishita N, Sasaki T, Nakazawa M, Kato Y, Yamadera T et al. (2007) Cytoplasmic destruction of $p 53$ by the endoplasmic reticulum-resident ubiquitin ligase 'Synoviolin'. EMBO J 26: 113-122.

39. Toledo F, Krummel KA, Lee CJ, Liu CW, Rodewald LW, Tang M et al. (2006) A mouse p53 mutant lacking the proline-rich domain rescues Mdm4 deficiency and provides insight into the Mdm2-Mdm4-p53 regulatory network. Cancer Cell 9: 273-285.

40. Itahana K, Mao H, Jin A, Itahana Y, Clegg HV, Lindström MS et al. (2007) Targeted inactivation of Mdm2 RING finger E3 ubiquitin ligase activity in the mouse reveals mechanistic insights into p53 regulation. Cancer Cell 12: 355-366.

41. Minsky N, Oren M (2004) The RING domain of Mdm2 mediates histone ubiquitylation and transcriptional repression. Mol Cell 16: 631-639.

42. Mendrysa SM, McElwee MK, Michalowski J, O'Leary KA, Young KM, Perry ME (2003) $\mathrm{mdm} 2$ Is critical for inhibition of p53 during lymphopoiesis and the response to ionizing irradiation. Mol Cell Biol 23: 462-472.

43. Terzian T, Wang Y, Van Pelt CS, Box NF, Travis EL, Lozano G (2007) Haploinsufficiency of Mdm2 and Mdm4 in tumorigenesis and development. Mol Cell Biol 27 5479-5485.

44. Alt JR, Greiner TC, Cleveland JL, Eischen CM (2003) Mdm2 haplo-insufficiency profoundly inhibits Myc-induced lymphomagenesis. EMBO J 22: 1442-1450.

45. Mendrysa O'Leary KA, McElwee MK, Michalowski J, Eisenman RN, Powell DA, Perry ME (2006) Tumor suppression and normal aging in mice with constitutively high p53 activity. Genes Dev 20: 16-21.

46. Bond GL, Hu W, Bond EE, Robins H, Lutzker SG, Arva NC et al. (2004) A single nucleotide polymorphism in the MDM2 promoter attenuates the p53 tumor suppressor pathway and accelerates tumor formation in humans. Cell 119: 591-602.

47. Ruijs MW, Schmidt MK, Nevanlinna H, Tommiska J, Aittomäki K, Pruntel R et al. (2007) The single-nucleotide polymorphism 309 in the MDM2 gene contributes to the Li-Fraumeni syndrome and related phenotypes. Eur J Hum Genet 15: 110-114.

48. Bougeard G, Baert-Desurmont S, Tournier I, Vasseur S, Martin C, Brugieres L et al. (2006) Impact of the MDM2 SNP309 and p53 Arg72Pro polymorphism on age of tumour onset in Li-Fraumeni syndrome. J Med Genet 43: 531-533.

49. Whibley C, Pharoah PD, Hollstein M (2009) p53 polymorphisms: cancer implications. Nat Rev Cancer 9: 95-107.

50. Rodriguez MS, Desterro JM, Lain S, Lane DP, Hay RT (2000) Multiple C-terminal lysine residues target p53 for ubiquitin-proteasome-mediated degradation. Mol Cell Biol 20 8458-8467.

51. Lohrum MA, et Woods DB, Ludwig RL, Bálint E, Vousden KH (2001) C-terminal ubiquitination of p53 contributes to nuclear export. Mol Cell Biol 21: 8521-8532.

52. Krummel KA, Lee CJ, Toledo F, Wahl GM (2005) The C-terminal lysines fine-tune P53 stress responses in a mouse model but are not required for stability control or transactivation. Proc Natl Acad Sci USA 102: 10188-10193.

53. Bossis G, Ferrara P, Acquaviva C, Jariel-Encontre I, Piechaczyk M (2003) c-Fos protooncoprotein is degraded by the proteasome independently of its own ubiquitinylation in vivo. Mol Cell Biol 23: 7425-7436.

54. Chen X, Chi Y, Bloecher A, Aebersold R, Clurman BE, Roberts JM (2004) N-acetylation and ubiquitin-independent proteasomal degradation of p21(Cip1). Mol Cell 16: 839-847.

55. Argentini M, Barboule N, Wasylyk B (2001) The contribution of the acidic domain of MDM2 to p53 and MDM2 stability. Oncogene 20: 1267-1275.

56. Kawai H, Wiederschain D, Yuan ZM (2003) Critical contribution of the MDM2 acidic domain to 553 ubiquitination. Mol Cell Biol 14: 4939-4947.

57. Meulmeester E, Frenk R, Stad R, de Graaf P, Marine JC, Vousden KH et al. (2003) Critical role for a central part of Mdm2 in the ubiquitylation of p53. Mol Cell Biol 14 4929-4938.

58. Fang S, Jensen JP, Ludwig RL, Vousden KH, Weissman AM (2000) Mdm2 is a RING finger-dependent ubiquitin protein ligase for itself and p53. J Biol Chem 275: 8945-8951. 
59. Uldrijan S, Pannekoek WJ, Vousden KH (2007) An essential function of the extreme Cterminus of MDM2 can be provided by MDMX. EMBO J 26: 102-112.

60. Poyurovsky MV, Priest C, Kentsis A, Borden KL, Pan ZQ, Pavletich N et al. (2007) The Mdm2 RING domain C-terminus is required for supramolecular assembly and ubiquitin ligase activity. EMBO J 26: 90-101

61. Kostic M, Matt T, Martinez-Yamout MA, Dyson HJ, Wright PE (2006) Solution structure of the $\mathrm{Hdm} 2 \mathrm{C} 2 \mathrm{H} 2 \mathrm{C} 4 \mathrm{RING}$, a domain critical for ubiquitination of p53. J Mol Biol 363 433-450.

62. Kubbutat MH, Ludwig RL, Ashcroft M, Vousden KH (1998) Regulation of Mdm2-directed degradation by the $\mathrm{C}$ terminus of p53. Mol Cell Biol 18: 5690-5698.

63. Maki CG (1999) Oligomerization is required for p53 to be efficiently ubiquitinated by MDM2. J Biol Chem 274: 16531-16535.

64. Linke K, Mace PD, Smith CA, Vaux DL, Silke J, Day CL (2008) Structure of the MDM2/ MDMX RING domain heterodimer reveals dimerization is required for their ubiquitylation in trans. Cell Death Differ 15: 841-848.

65. Thrower JS, Hoffman L, Rechsteiner M, Pickart CM (2000) Recognition of the polyubiquitin proteolytic signal. EMBO J 19: 94-102.

66. Hicke L, Dunn R (2003) Regulation of membrane protein transport by ubiquitin and ubiquitin-binding proteins. Annu Rev Cell Dev Biol 19: 141-172. Review.

67. Li M, Brooks CL, Wu-Baer F, Chen D, Baer R, Gu W (2003) Mono- versus polyubiquitination: differential control of p53 fate by Mdm2. Science 302: 1972-1975.

68. Stommel JM, Marchenko ND, Jimenez GS, Moll UM, Hope TJ, Wahl GM (1999) A leucine-rich nuclear export signal in the p53 tetramerization domain: regulation of subcellular localization and p53 activity by NES masking. EMBO J 18: 1660-1672.

69. Brooks CL, Gu W. p53 ubiquitination: Mdm2 and beyond. Mol Cell 2006; 21: 307-315. Review.

70. Carter S, Bischof O, Dejean A, Vousden KH (2007) C-terminal modifications regulate MDM2 dissociation and nuclear export of p53. Nat Cell Biol 9: 428-435.

71. Sasaki M, Nie L, Maki CG (2007) MDM2 binding induces a conformational change in p53 that is opposed by heat-shock protein 90 and precedes p53 proteasomal degradation. $J$ Biol Chem 282: 14626-14634.

72. Nie L, Sasaki M, Maki CG. Regulation of p53 nuclear export through sequential changes in conformation and ubiquitination. J Biol Chem 2007; 282: 14616-14625.

73. Grossman SR, Deato ME, Brignone C, Chan HM, Kung AL, Tagami H et al. (2003) Polyubiquitination of $p 53$ by a ubiquitin ligase activity of p300. Science 300 : 342-344.

74. Sherr CJ (2006) Divorcing ARF and p53: an unsettled case. Nat Rev Cancer 6: 663-673

75. Marine JC, Dyer MA, Jochemsen AG (2007) MDMX: from bench to bedside. J Cell Sci 120: 371-378. Review.

76. Lu X, Ma O, Nguyen TA, Jones SN, Oren M, Donehower LA (2007) The Wip1 Phosphatase acts as a gatekeeper in the p53-Mdm2 autoregulatory loop. Cancer Cell 12 342-354

77. Fiscella M, Zhang H, Fan S, Sakaguchi K, Shen S, Mercer WE et al. (1997) Wip1, a novel human protein phosphatase that is induced in response to ionizing radiation in a p53 dependent manner. Proc Natl Acad Sci USA 94: 6048-6053.

78. Rossi M, Demidov ON, Anderson CW, Appella E, Mazur SJ (2008) Induction of PPM1D following DNA-damaging treatments through a conserved p53 response element coincides with a shift in the use of transcription initiation sites. Nucleic Acids Res $\mathbf{3 6}$ $7168-7180$

79. Maya R, Balass M, Kim ST, Shkedy D, Leal JF, Shifman $O$ et al. (2001) ATM-dependent phosphorylation of Mdm2 on serine 395: role in p53 activation by DNA damage. Genes Dev 15: 1067-1077.

80. Yu E, Ahn YS, Jang SJ, Kim MJ, Yoon HS, Gong G et al. (2007) Overexpression of the wip1 gene abrogates the p38 MAPK/p53/Wip1 pathway and silences p16 expression in human breast cancers. Breast Cancer Res Treat 101: 269-278.

81. Batchelor E, Mock CS, Bhan I, Loewer A, Lahav G (2008) Recurrent initiation: a mechanism for triggering p53 pulses in response to DNA damage. Mol Cell 30: 277-289.

82. Abe Y, Oda-Sato E, Tobiume K, Kawauchi K, Taya Y, Okamoto K et al. (2008) Hedgehog signaling overrides p53-mediated tumor suppression by activating Mdm2. Proc Natl Acad Sci USA 105: 4838-4843.

83. Meek DW, Knippschild U (2003) Posttranslational modification of MDM2. Mol Cancer Res 1: 1017-1026. Review.

84. Chiu SY, Asai N, Costantini F, Hsu W (2008) SUMO-specific protease 2 is essential for modulating p53-Mdm2 in development of trophoblast stem cell niches and lineages. PLOS Biol 6: e310.

85. Roegiers F, Jan YN (2004) Asymmetric cell division. Curr Opin Cell Biol 16: 195-205. Review.

86. Juven-Gershon T, Shifman O, Unger T, Elkeles A, Haupt Y, Oren M (1998) The Mdm2 oncoprotein interacts with the cell fate regulator Numb. Mol Cell Biol 18: 3974-3982.

87. Yogosawa S, Miyauchi Y, Honda R, Tanaka H, Yasuda H (2003) Mammalian Numb is a target protein of Mdm2, ubiquitin ligase. Biochem Biophys Res Commun 302: 869-872.

88. Colaluca IN, Tosoni D, Nuciforo P, Senic-Matuglia F, Galimberti V, Viale G et al. (2008) NUMB controls p53 tumour suppressor activity. Nature 451: 76-80.

89. Sherley JL, Stadler PB, Johnson DR (1995) Expression of the wild-type p53 antioncogene induces guanine nucleotide-dependent stem cell division kinetics. Proc Natl Acad Sci USA 92: $136-140$

90. Sui G, Affar el B, Shi Y, Brignone C, Wall NR, Yin P et al. (2004) Yin Yang 1 is a negative regulator of p53. Cell 117: 859-872.
91. Chen D, Zhang J, Li M, Rayburn ER, Wang H, Zhang R (2009) RYBP stabilizes p53 by modulating MDM2. EMBO Rep 10: 166-172.

92. Chang YC, Lee YS, Tejima T, Tanaka K, Omura S, Heintz NH et al. (1998) mdm2 and bax, downstream mediators of the p53 response, are degraded by the ubiquitinproteasome pathway. Cell Growth Differ 9: 79-84

93. Honda R, Yasuda $\mathrm{H}$ (2000) Activity of MDM2, a ubiquitin ligase, toward $p 53$ or itself is dependent on the RING finger domain of the ligase. Oncogene 19: 1473-1476.

94. Stommel JM, Wahl GM (2004) Accelerated MDM2 auto-degradation induced by DNA-damage kinases is required for p53 activation. EMBO J 23: 1547-1556.

95. Clegg HV, Itahana K, Zhang Y (2008) Unlocking the Mdm2-p53 loop: ubiquitin is the key. Cell Cycle 7: 287-292

96. Linares LK, Kiernan R, Triboulet R, Chable-Bessia C, Latreille D, Cuvier $O$ et al. (2007) Intrinsic ubiquitination activity of PCAF controls the stability of the oncoprotein Hdm2. Nat Cell Biol 9: 331-338.

97. Weber JD, Taylor LJ, Roussel MF, Sherr CJ, Bar-Sagi D (1999) Nucleolar Arf sequesters Mdm2 and activates p53. Nat Cell Biol 1: 20-26.

98. Kurki S, Peltonen K, Latonen L, Kivihariu TM, Ojala PM, Meek D et al. (2004) Nucleola protein NPM interacts with HDM2 and protects tumor suppressor protein p53 from HDM2mediated degradation. Cancer Cell 5: 465-475.

99. Wei X, Yu ZK, Ramalingam A, Grossman SR, Yu JH, Bloch DB et al. (2003) Physical and functional interactions between PML and MDM2. J Biol Chem 278: 29288-29297.

100. Bernardi R, Scaglioni PP, Bergmann S, Horn HF, Vousden KH, Pandolfi PP et al. (2004) PML regulates p53 stability by sequestering Mdm2 to the nucleolus. Nat Cell Biol 6: $665-672$.

101. Lindström MS, Deisenroth C, Zhang $Y$ (2007) Putting a finger on growth surveillance: insight into MDM2 zinc finger-ribosomal protein interactions. Cell Cycle 6: 434-437. Review.

102. Horn HF, Vousden KH (2008) Cooperation between the ribosomal proteins L5 and L11 in the p53 pathway. Oncogene 27: $5774-5784$

103. Opferman JT, Zambetti GP (2006) Translational research? Ribosome integrity and a new p53 tumor suppressor checkpoint. Cell Death Differ 3: 898-901. Review.

104. Marine JC, Jochemsen AG (2005) Mdmx as an essential regulator of p53 activity. Biochem Biophys Res Commun 331: 750-760.

105. Wade M, Wahl GM (2009) Targeting Mdm2 and Mdmx in cancer therapy: better living through medicinal chemistry? Mol Cancer Res 7: 1-11.

106. Terzian T, Suh YA, Iwakuma T, Post SM, Neumann M, Lang GA et al. (2008) The inherent instability of mutant $\mathrm{p} 53$ is alleviated by Mdm2 or p16INK4a loss. Genes Dev 22: 1337-1344.

107. Shikama N, Lee CW, France S, Delavaine L, Lyon J, Krstic-Demonacos M et al. (1999) A novel cofactor for p300 that regulates the p53 response. Mol Cell 4: 365-376

108. Coutts AS, Boulahbel H, Graham A, La Thangue NB (2007) Mdm2 targets the p53 transcription cofactor JMY for degradation. EMBO Rep 8: 84-90.

109. Moumen A, Masterson P, O'Connor MJ, Jackson SP (2005) hnRNP K: an HDM2 target and transcriptional coactivator of $\mathrm{p} 53$ in response to DNA damage. Cell 123: 1065-1078.

110. Boesten LS, Zadelaar SM, De Clercq S, Francoz S, van Nieuwkoop A, Biessen EA et al. (2006) Mdm2, but not Mdm4, protects terminally differentiated smooth muscle cells from p53-mediated caspase-3-independent cell death. Cell Death Differ 13: 2089-2098.

111. D’Orazi G, Cecchinelli B, Bruno T, Manni I, Higashimoto Y, Saito S et al. (2002) Homeodomain-interacting protein kinase-2 phosphorylates p53 at Ser 46 and mediates apoptosis. Nat Cell Biol 4: 11-19.

112. Rinaldo C, Prodosmo A, Mancini F, lacovelli S, Sacchi A, Moretti F et al. (2007) MDM2regulated degradation of HIPK2 prevents p53Ser46 phosphorylation and DNA damageinduced apoptosis. Mol Cell 25: 739-750.

113. Yang JY, Zong CS, Xia W, Yamaguchi H, Ding Q, Xie X et al. (2008) ERK promotes tumorigenesis by inhibiting FOXO3a via MDM2-mediated degradation. Nat Cell Biol 10: 138-148.

114. Maguire M, Nield PC, Devling T, Jenkins RE, Park BK, Polañski R et al. (2008) MDM2 regulates dihydrofolate reductase activity through monoubiquitination. Cancer Res 68 : 3232-3242.

115. Vazquez A, Bond EE, Levine AJ, Bond GL (2008) The genetics of the p53 pathway, apoptosis and cancer therapy. Nat Rev Drug Discov 7: 979-987. Review.

116. Oliner JD, Kinzler KW, Meltzer PS, George DL, Vogelstein B (1992) Amplification of a gene encoding a p53-associated protein in human sarcomas. Nature 358: 80-83.

117. Valentin-Vega YA, Barboza JA, Chau GP, El-Naggar AK, Lozano G (2007) High levels of the p53 inhibitor MDM4 in head and neck squamous carcinomas. Hum Pathol 38: 1553-1562.

118. Cordon-Cardo C, Latres E, Drobnjak M, Oliva MR, Pollack D, Woodruff JM et al. (1994) Molecular abnormalities of mdm2 and p53 genes in adult soft tissue sarcomas. Cancer Res 54: 794-799.

119. Watanabe T, Hotta T, Ichikawa A, Kinoshita T, Nagai H, Uchida T et al. (1994) The MDM2 oncogene overexpression in chronic lymphocytic leukemia and low-grade lymphoma of $B$ cell origin. Blood 84: 3158-3165

120. Jones SN, Hancock AR, Vogel H, Donehower LA, Bradley A (1998) Overexpression of Mdm2 in mice reveals a p53-independent role for Mdm2 in tumorigenesis. Proc Natl Acad Sci USA 95: 15608-15612.

121. Lundgren $K$, Montes de Oca Luna $R$, McNeill $Y B$, Emerick EP, Spencer $B$, Barfield CR et al. (1997) Targeted expression of MDM2 uncouples S phase from 
mitosis and inhibits mammary gland development independent of p53. Genes Dev 11 : 714-725.

122. Bouska A, Lushnikova T, Plaza S, Eischen CM (2008) Mdm2 promotes genetic instability and transformation independent of p53. Mol Cell Biol 28: 4862-4874.

123. Froment $P$, Dupont $J$, Christophe-Marine $J(2008)$ Mdm2 exerts pro-apoptotic activities by antagonizing insulin-like growth factor-I-mediated survival. Cell Cycle 7: 3098-3103.

124. Girnita L, Girnita A, Larsson O (2003) Mdm2-dependent ubiquitination and degradation of the insulin-like growth factor 1 receptor. Proc Natl Acad Sci USA 100: 8247-8252.

125. Martins CP, Brown-Swigart L, Evan Gl (2006) Modeling the therapeutic efficacy of p53 restoration in tumors. Cell 127: 1323-1334.

126. Ventura A, Kirsch DG, McLaughlin ME, Tuveson DA, Grimm J, Lintault $L$ et al. (2007) Restoration of p53 function leads to tumour regression in vivo. Nature 445: 661-665.
127. Xue W, Zender L, Miething C, Dickins RA, Hernando E, Krizhanovsky V et al. (2007) Senescence and tumour clearance is triggered by p53 restoration in murine live carcinomas. Nature 445: 656-660.

128. Issaeva N, Bozko P, Enge M, Protopopova M, Verhoef LG, Masucci M et al. (2004) Small molecule RITA binds to p53, blocks p53-HDM-2 interaction and activates p53 function in tumors. Nat Med 10: 1321-1328.

129. Vassilev LT, Vu BT, Graves B, Carvajal D, Podlaski F, Filipovic $Z$ et al. (2004) In vivo activation of the p53 pathway by small-molecule antagonists of MDM2. Science 303 844-848.

130. Yang Y, Ludwig RL, Jensen JP, Pierre SA, Medaglia MV, Davydov IV et al. (2005) Small molecule inhibitors of HDM2 ubiquitin ligase activity stabilize and activate p53 in cells. Cancer Cell 7: 547-559. 